\title{
Deep Neural Networks Based on Transfer Learning Approaches to Classification of Gun and Knife Images
}

\author{
(iD)Mehmet Tevfik Ağdaş ${ }^{1}$, (D) Muammer Türkoğlu², (DS Sevinç Gülseçen ${ }^{3}$ \\ ${ }^{1}$ Department of Electronic and Automation, Artvin Çoruh University, Artvin, Turkey; \\ mtevfikagdas@artvin.edu.tr; \\ ${ }^{2}$ Corresponding Author; Department of Computer Engineering, Bingol University, Bingöl, Turkey; \\ mturkoglu@bingol.edu.tr; \\ ${ }^{3}$ Informatics Department, Istanbul University, Istanbul, Turkey; gulsecen@istanbul.edu.tr
}

Received 4 March 2021; Revised 15 March 2021; Accepted 16 March 2021; Published online 25 March 2021

\begin{abstract}
Most of the criminal acts are performed using criminal tools. One of the most effective ways of preventing crime is to observe and detect offensive weapons by security camera systems. Deep learning techniques can show very high-performance in observing and perceiving objects. In the current study, the performances of the pre-trained AlexNet, VGG16, and VGG19 models based on convolutional neural networks, were tested for the detection and classification of criminal tools such as guns and knives. In the study, the training process was carried out using transfer learning approaches such as Fine-tuning and Training from scratch based on deep architectures. To test the deep architectures used in the proposed study, the gun and knife datasets frequently used in the literature were collected and combined with new datasets obtained originally from search engines and videos, and then their performances were tested. In the experimental results, the VGG16 model based on fine-tuning for the two and three classes achieved the highest accuracy in detecting criminal devices with a rate of $99.73 \%$ and $99.67 \%$, respectively. As a result, the study has observed that offensive weapons could be detected with security cameras using learned weights of deep architectures.
\end{abstract}

Keywords: Pattern recognition, Gun and Knife Detection, Deep Learning, Offensive weapons, Convolutional Neurol Network

\section{Introduction}

Globally the number of violent incidents committed by individuals with their weapons is increasing day by day. In the 2019 report named the Map of the Armed Violence in Turkey released by Umut Foundation, an institution standing against the individual armament in Turkey, it has been stated that the number of violent events committed by using personal arms is arising every passing year in Turkey. The report has declared that individuals involving criminal acts in 2019 took their places in the media for 3623 disagreements. Out of these events, firearms were used in 2867 conflicts, and all kinds of sharp and piercing tools were used in the remaining 756 incidents. Besides, according to the foundation's research results obtained from other sources, it has been stated that 2,211 people died, and 3,736 people were injured in the same year resulting from firearms and sharp objects utilization [1].

The Umut Foundation report also includes a comparative regional armed violence report for the years 2015-2019. As seen in Figure 1, at least a 46\% increase in the numbers of violent crimes and crime tools is observed in every region of Turkey between 2015 and 2019 years [1]. That people openly carry offensive instruments is a sign of possible acts of violence. Detection of crime weapons may prevent violent incidents. Governments prohibit carrying offensive weapons with sanctions, and besides, assign law enforcement officers to detect. Law enforcement officers make observations directly in public areas and also with security camera systems through computers.

Object detection and recognition from images or videos have recently become one of the most popular research/application topics. Various applications such as recognitions and detections of face, vehicle, plant, license plate, item are performed automatically. The security sector is one of the most prominent areas that object detection or recognition applications can have significant effects. Today, security cameras are used in many living areas, and images taken from these security cameras play a primary 


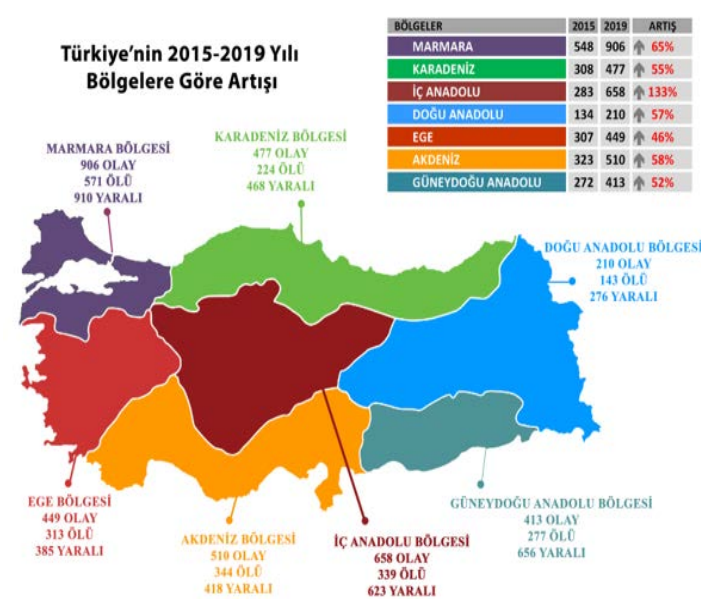

Figure 1. Umut Foundation 2015-2019 Armed Violence by Regions [1].

role in solving many incidents and providing information for forensic channels [2]. Usually, the usage of security cameras and getting crucial information for public safety are conducted by law enforcement. However, using computer vision systems for object perception or detection in today's technology will ensure reliability in the detection and reduce the law enforcement officers' workloads. Besides, since the computers carry out the automatic object detection and recognition processes, the law enforcement officers can get the information required more quickly and accurately. Research has shown that law enforcement officers' prompt intervention in preventing crime is of great importance [3].

In the method's selection for this study, some studies on gun and knife detection and perception in the literature were examined. In their studies, Garega et al. performed feature extraction from MPEG7 videos and made classifications using SVM algorithms to detect guns and knives through security cameras [4]. Gonzalez et al. conducted a real-time gun detection study in the closed-circuit security camera (CCTV) system. They used R-CNN based pre-trained ResNet-50 architecture [5]. Verma and Dhillon conducted a study for the detection of handheld guns by using Faster R-CNN, which is one of the deep learning techniques. They used Deep Convolution Network (DCN) for automatic gun detection from scattered scenes [6]. Tiwari and Verma carried out a computer vision-based visual gun detection study using a Harris point of interest detector. They have extracted the unrelated object from the images using the K-Means algorithm. They made use of the color-based sections in the appearances [7]. Kmiec et al. presented a new application of Active View Patterns from Computer vision techniques to detect the blades in images. In the examination, they sought an answer to whether there was a knife in the security camera images [8]. Castillo et al. made a preprocessing through the brightness guidance for automatic cold steel gun detection in security camera surveillance videos featured with deep learning. They used CNN-based ResNet architectures for detection. They also used the DaCoLT (Darkening and Contrast in Learning and Testing stages) technique for brightness-guided preprocessing [9]. Carrobles et al. conducted a Faster R-CNN-based gun and knife detection study for video surveillance. They used R-CNN based SqueezeNet architecture and GoogleNet architecture in their studies. In test measurements of the detection process, they achieved a performance score of $46.68 \%$ with GoogleNet architecture and $85.44 \%$ with SqueezeNet architecture [10]. Jain et al. conducted a gun detection study using artificial intelligence and deep learning techniques for security applications. They used the RCNN neural network SSD VGG-16 architecture in their work and achieved an average performance score of $73.8 \%$ [11]. Olmos et al. made automatic gun detection in videos using deep learning approaches. They used an open-access dataset. In their studies, they performed the training process with the CNN based R-CNN model [12].

In this study, the pre-trained deep architecture performance was evaluated to classify guns, knives, and regular images. In this direction, approaches such as transfer learning and scratch were used for pretrained deep architectures. In the experimental studies, a comprehensive dataset containing guns, knives, and ordinary images was used to test the proposed system. In the experimental results, VGG16 architecture achieved a criminal devices recognition performance of $99.73 \%$. 
A $\breve{g} d a s ̧$ et al.

The present study was carried out to automatically detect guns and knives that could be used in violent incidents. For this purpose, deep learning techniques were suggested. The measurements were made on high-performance GPU supported computers with AlexNet, Vgg16, and Vgg19 architectures previously trained with deep learning techniques. Previous object detection studies in the literature were examined, and the existing datasets were analyzed. To increase the dataset reliability, original and new gun and knife data were collected from internet pages, combined with the existing dataset, and tested with architectures. The architectures of the dataset used, and the test performances have been compared with the table. From the current study results in the literature, methods with better performance have been recommended for applications that require this type of perception.

The main contributions of this study are given below:

- In experimental studies, it has been determined that the learned weights of pre-trained Deep architectures provide high performance in detecting crime instruments.

- In this study, the performances of deep architectures such as AlexNet, VGG16, and VGG19 were evaluated, and their strengths were demonstrated.

- The proposed study can be used in simple and real-time applications.

\section{Material and Method}

In this paper, we evaluated performances of deep models based on transfer learning approaches to the classification of gun and knife images. The general flow diagram of the proposed study is given in Figure 2.

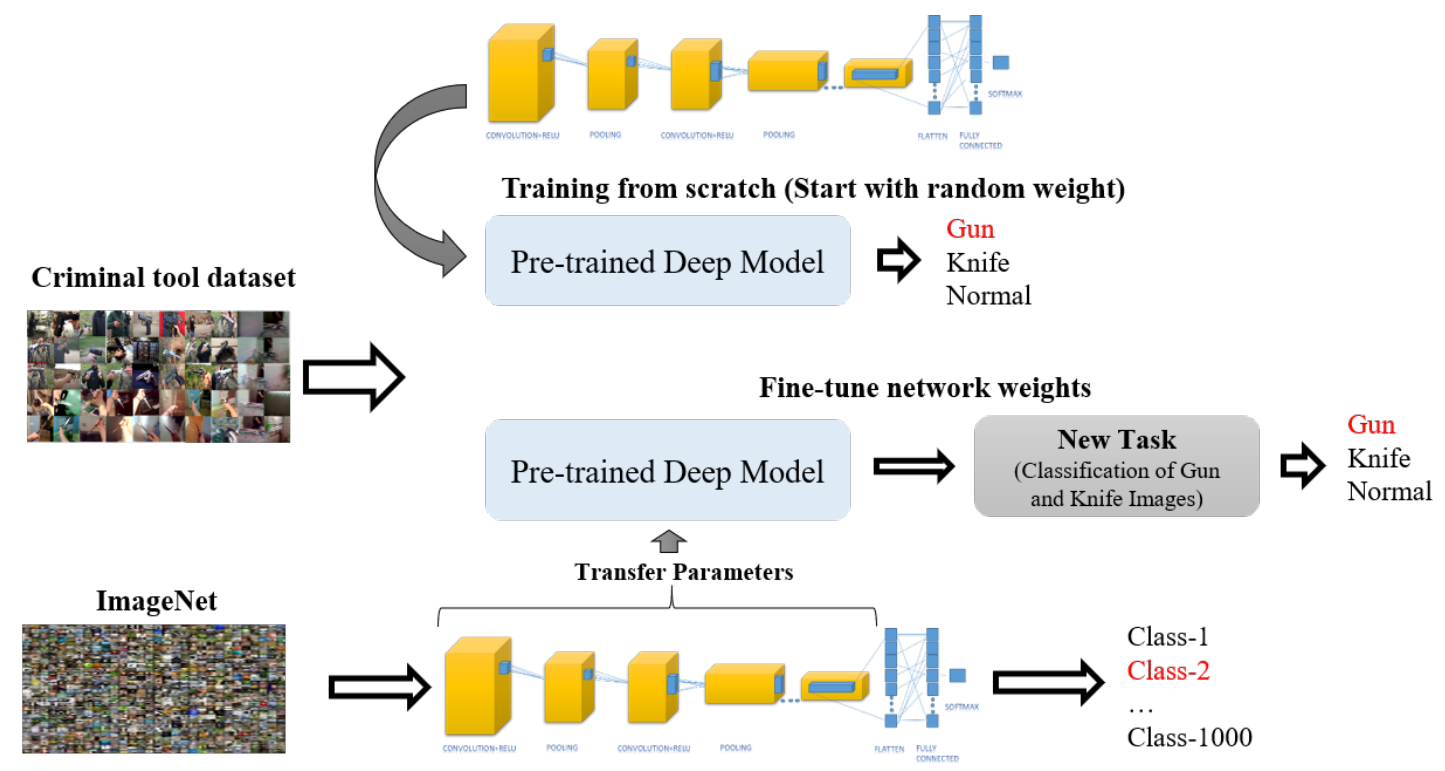

Figure 2. The general structure of the proposed study

\subsection{Dataset}

In this study, a data set suitable for the purposes was created by collecting images from open access data sets $[9,12-14]$, which have repeatedly been the source of many previous studies in the literature and original internet browsers and video pages [4, 15]. This dataset has 16000 images containing 9500 knives, 3500 guns, and 3000 ordinary pictures. Sample images belonging to these classes are given in Figure 3. 

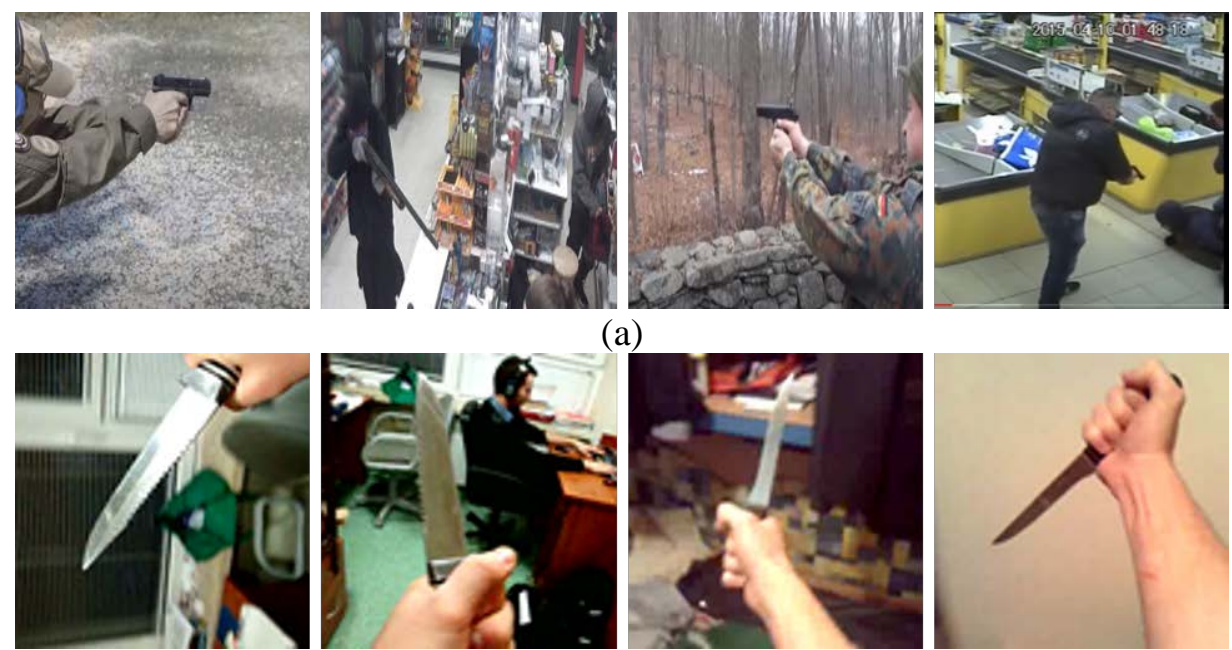

(a)
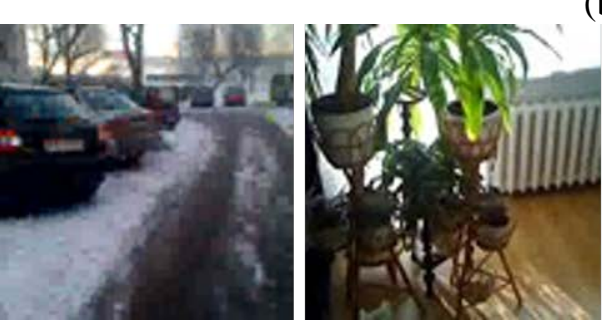

(b)
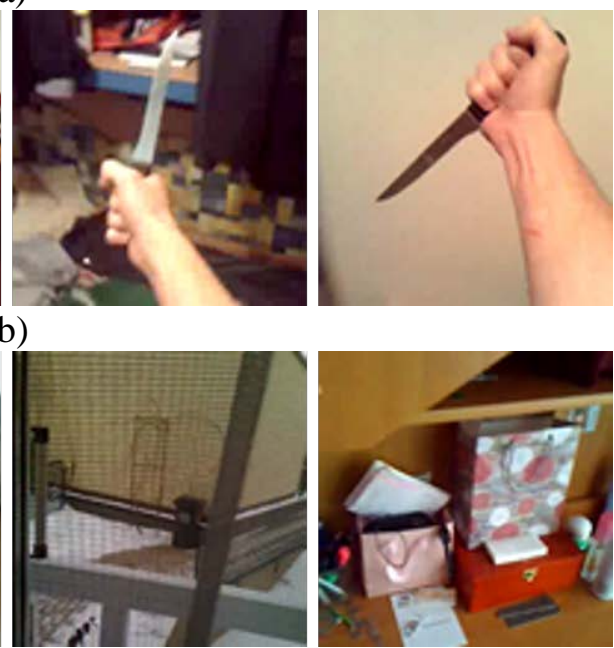

(c)

Figure 3. Data set example, a) Gun images, b) Knife images, c) Normal images

\subsection{Convolutional Neural Networks}

A convolutional neural network (CNN) is a neural network that has matrix multiplication and convolution system in at least one of its layers. CNNs must have at least one or more of the convolution layer, non-linearity layer, pooling layer, fully connected layer, and hidden layers (Figure 4).

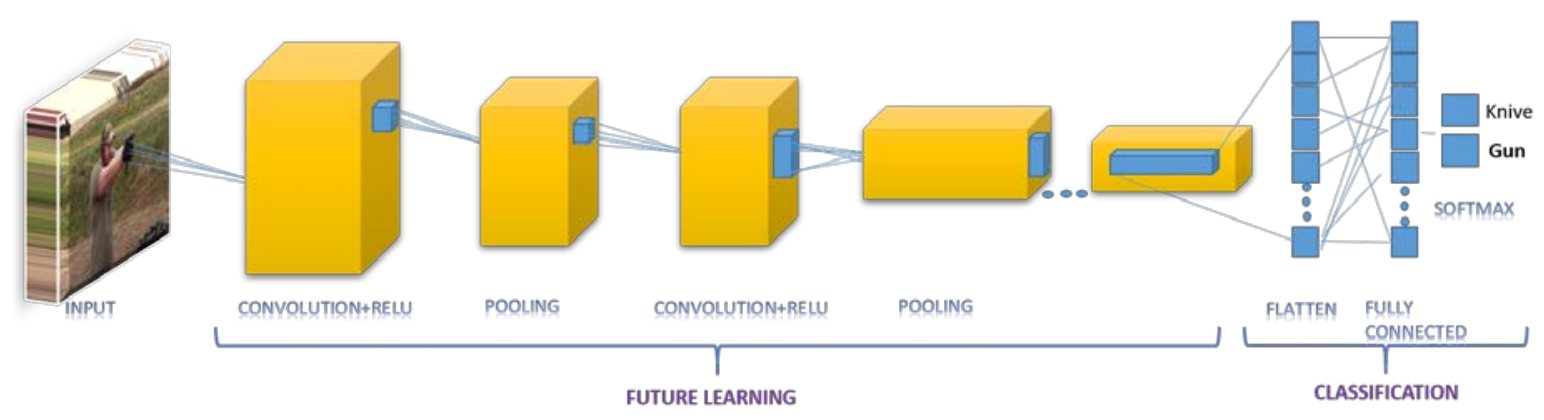

Figure 4. Convolutional Neural Network Layers

The layers given in Figure 4 can be briefly summarized as follows [16-19]:

- Convolutional Layer- The layer used to detect properties,

- Non-Linearity Layer- The layer used to make the system nonlinear,

- Pooling Layer- The layer controlling the number of weights and over-fitting,

- Flattening Layer- Data preparation layer for classical neural network,

- Fully Connected Layer- Standard neural network layer used for classification. 
A $\breve{g} d a s ̧$ et al.

CNNs are inspired by people's ability to comprehend events. It is in the deep feedforward artificial neural networks class to analyze images. CNN uses 2D and 3D images when generating spatial and configuration information. Convolutional neural networks use three receptive fields, shared weights, and sub-sampling mechanisms to increase the models' working areas. CNNs are generally used to recognize patterns in images. For this reason, features within images can be encoded into architectures, thus making them more suitable for vision network-oriented tasks while further reducing the parameters required for model building. CNN applications are widely used for object detection, prediction, text detection, image detection, etc. [18-24].

\subsection{Deep Architectures}

In this study, the convolutional neural network models AlexNet, VGG-16, and VGG-19 architectures, which were previously trained on over one million images, were used, and the comparison results were obtained.

\subsubsection{AlexNet Architecture}

AlexNet [25] is a convolutional neural network architecture created in 2012. It has 60 million parameters and 650 million neurons. AlexNet comprises five Convolutional Layers and three Fully Connected Layers. The Multiple Convolutional Kernels layer (Filters layer) discerns unusual features in an image. There are usually plenty of same-size cores in a single convolution layer. For example, AlexNet's first Conv Layer contains 96 cores at 11x11x3. The width and height of the cores are usually the same sizes. The Max Pooling layer comes after the first two-convolutional layers. The third, fourth and fifth convolutional layers directly connect. The Overlapping Max Pooling layer, whose output goes to a series of two fully connected layers, follows the fifth convolutional layer. The fully connected second layer is followed by a 1000 class labeled Softmax classifier [26-27]. For the Alexnet architecture, images should be specified as 256x256 inputs. The structure of the AlexNet architecture given in Figure 5.

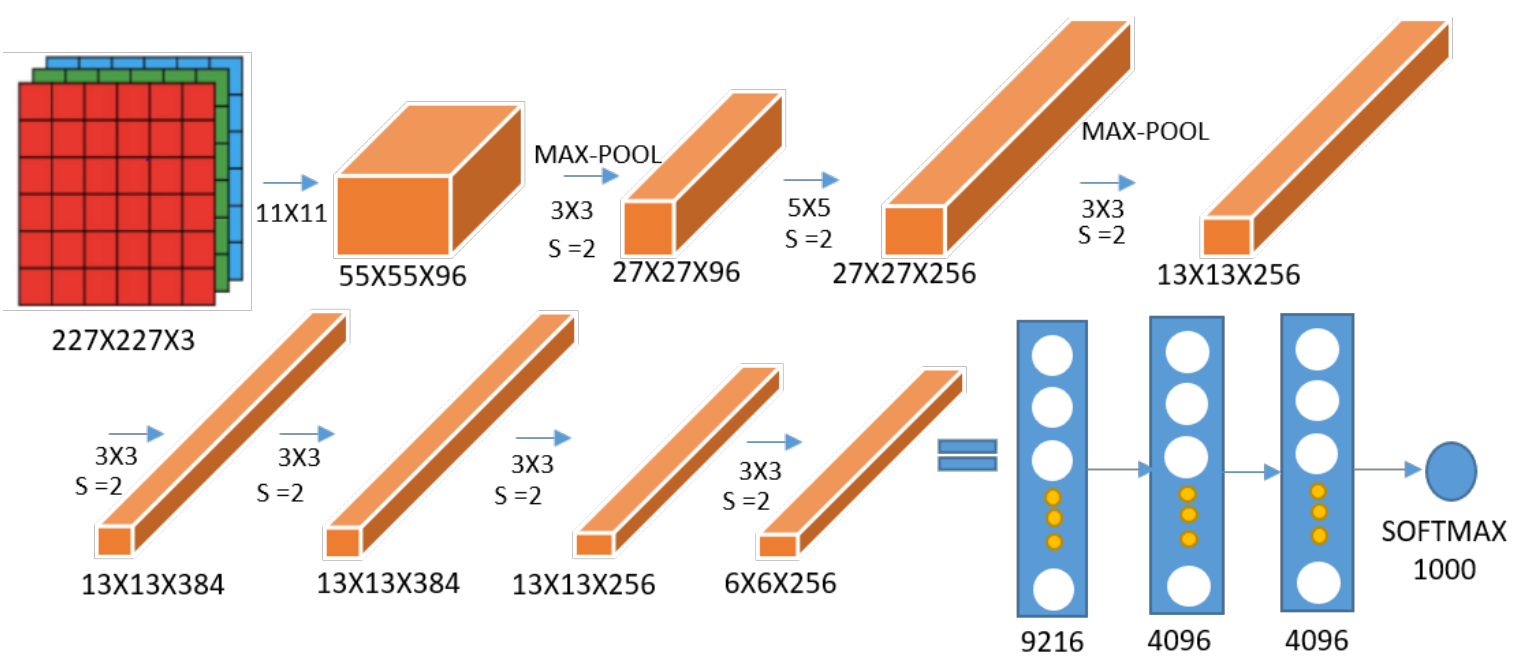

Figure 5. AlexNet Architecture

\subsubsection{VGG Architecture}

The VGG-16 architecture [28], including 21 main layers, consists of convolutional, pooling, and fully connected layers. Its architecture has an increasing network structure. The image input resolution must be 224x224 pixels in size. In this architecture, the last layers comprise fully connected layers used for feature extraction [29-30]. The structure of the VGG16 architecture given in Figure 6. 

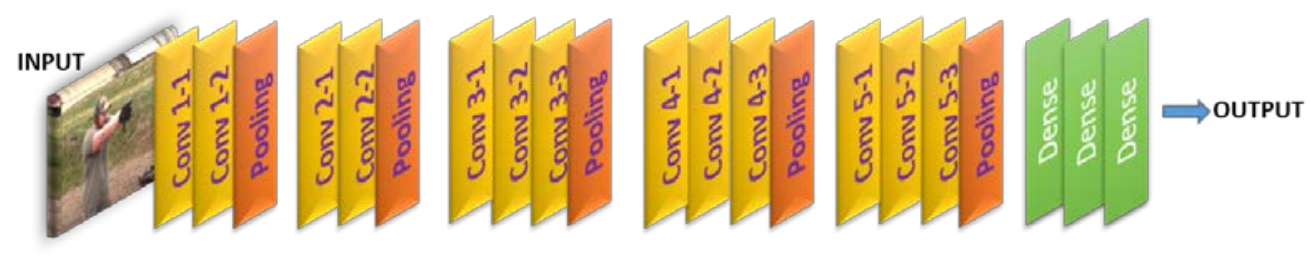

Figure 6. VGG16 Architecture

VGG-19 architecture [28] with 24 main layers consists of 16 convolutional, five pooling, and three fully connected layers. Since VGG-19 has a deep network, the filters are used in the convolution layer to reduce the parameters' number. In this architecture, the image input size is 3x3 pixels. The VGG-19 architecture contains nearly 138 million parameters [29-30]. The structure of the VGG19 architecture given in Figure 7.

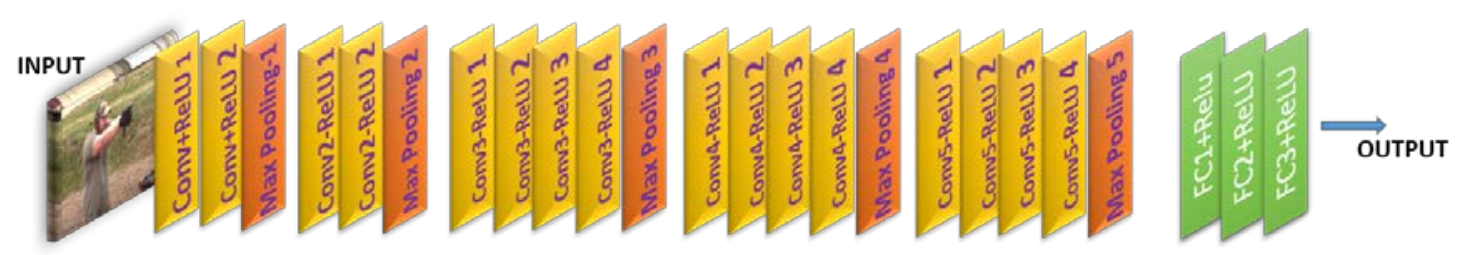

Figure 7. VGG19 Architecture

\subsection{Transfer learning approaches}

Pre-trained deep models contain parameters that have been trained using a large multi-class data set. Two transfer learning approaches, Fine-tuning and Training from scratch are available to use these deep models in other data sets. The fine-tuning method uses the learned weights of pre-trained deep models. This approach is based on adapting deep architectures by replacing the last three layers with new three layers to solve another classification problem. On the other hand, the Training from scratch approach uses the pre-trained model architecture to train the dataset accordingly. The starting weights of the model begin randomly [21, 31].

\section{Experimental Works}

The experimental studies were performed using MATLAB (R2020a) software on a computer equipped with RTX 2080 GPU card, 32 GB Ram, and Intel Core i7. In experimental studies, 2-class and 3-class data sets were used. These data sets were split into training and test sets, at the ratios of $80-20 \%$, 60$40 \%, 40-60 \%$, and $20-80 \%$, and a comprehensive experimental study was conducted for each. Besides, the network parameters used for training the pre-trained ESA architectures used in this study are given in Table 1.

Table 1 The deep network parameters used in the current study.

\begin{tabular}{|l|l|}
\hline Mini-batch size & $8-16$ \\
\hline Maximum epoch number & $5-20$ \\
\hline Weight decay factor & 0.01 \\
\hline Initial learning rate & 0.0001 \\
\hline Optimization method & $\begin{array}{l}\text { SGDM (Stochastic Gradient Descent } \\
\text { with Momentum) }\end{array}$ \\
\hline
\end{tabular}

Accuracy scores obtained in the experimental study performed for the 2-class (knife and normal) data set are given in Table 2 . 
Table 2 Accuracy scores (\%) for the 2-class data set

\begin{tabular}{|l|l|l|l|l|l|c|}
\hline Approaches & Models & $\mathbf{8 0 - 2 0}$ & $\mathbf{6 0 - 4 0}$ & $\mathbf{4 0 - 6 0}$ & $\mathbf{2 0 - 8 0}$ & Average \\
\hline \multirow{3}{*}{ Fine-tuning } & Alexnet & 98.80 & 98.31 & 97.39 & 96.48 & 97.74 \\
\cline { 2 - 7 } & VGG16 & 99.73 & 99.65 & 99.42 & 98.77 & 99.38 \\
\cline { 2 - 7 } & VGG19 & 99.65 & 99.53 & 99.25 & 98.66 & 99.27 \\
\hline \multirow{2}{*}{$\begin{array}{l}\text { Training from } \\
\text { scratch }\end{array}$} & Alexnet & 87.87 & 87.09 & 84.05 & 79.40 & 84.58 \\
\cline { 2 - 7 } & VGG16 & 84.73 & 83.57 & 80.23 & 74.38 & 80.72 \\
\cline { 2 - 7 } & VGG19 & 83.76 & 79.46 & 77.46 & 75.40 & 79.02 \\
\hline
\end{tabular}

In Table 2, separation values for four different training/test clusters are used, and separate results are given for each. According to these results, among deep models based on the fine-tuning approach, the VGG16 architecture achieved the highest accuracy on average, while the AlexNet architecture showed the lowest performance. On the other hand, among deep models based on the training from scratch approach, the Alexnet architecture obtained the highest accuracy on average, while the VGG19 architecture achieved the lowest performance. Additionally, the VGG16 architecture based on the Finetuning approach obtained the highest accuracy score among deep models with a 99.73\% success for separating training/testing clusters as $80-20$, while an $87.87 \%$ score was obtained with the AlexNet architecture based on the training from scratch approach. The confusion matrices of the highest performance based on these fine-tuning and the training from scratch approaches are given in Figure 8.

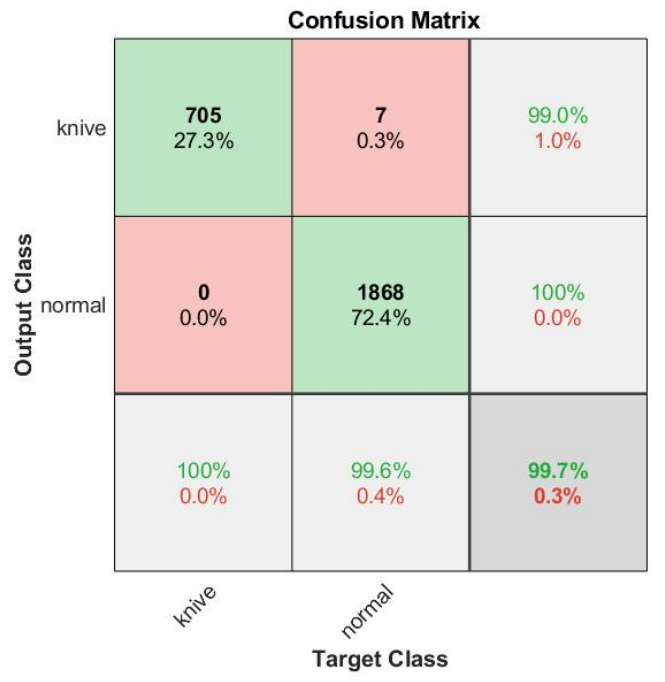

(a)

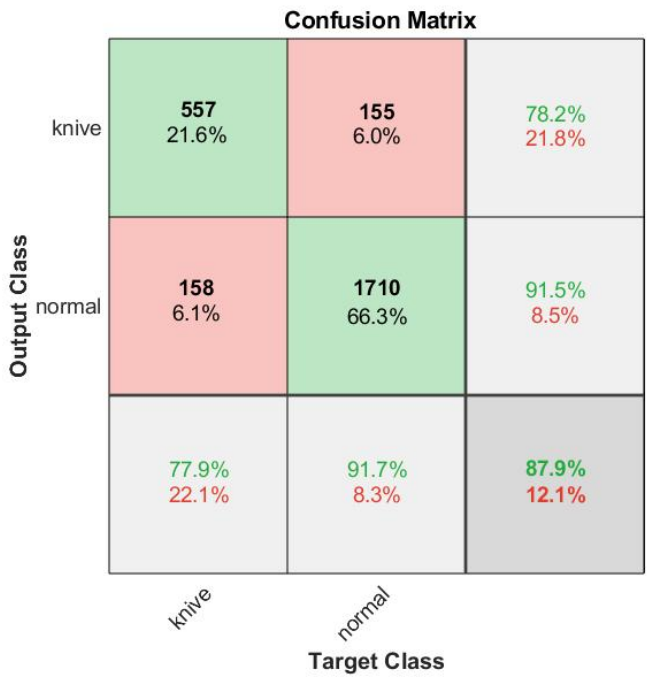

(b)

Figure 8. Confusion Matrices of proposed deep model based approaches to 2-class, a) Fine-tuning, b) Training from scratch.

The accuracy scores obtained in the experimental studies performed for the 3-class (knife, gun, and ordinary) data set are given in Table 3.

Table 3 Accuracy scores (\%) for the 3-class data set

\begin{tabular}{|l|l|l|l|l|l|c|}
\hline Approaches & Models & $\mathbf{8 0 - 2 0}$ & $\mathbf{6 0 - 4 0}$ & $\mathbf{4 0 - 6 0}$ & $\mathbf{2 0 - 8 0}$ & Average \\
\hline \multirow{3}{*}{ Fine-tuning } & Alexnet & 98.86 & 98.68 & 97.82 & 96.72 & 98.02 \\
\cline { 2 - 7 } & VGG16 & 99.62 & 99.41 & 99.29 & 98.64 & 99.24 \\
\cline { 2 - 7 } & VGG19 & 99.59 & 99.27 & 99.15 & 98.59 & 99.15 \\
\hline \multirow{2}{*}{$\begin{array}{l}\text { Training } \\
\text { from scratch }\end{array}$} & Alexnet & 86.77 & 85.25 & 80.48 & 72.86 & 81.34 \\
\cline { 2 - 7 } & VGG16 & 85.74 & 83.31 & 78.18 & 70.03 & 79.31 \\
\cline { 2 - 7 } & VGG19 & 83.67 & 79.69 & 74.04 & 64.64 & 75.51 \\
\hline
\end{tabular}

As seen from Table 7, the average highest accuracy among deep models based on the Fine-tuning approach was achieved as $99.24 \%$ with the VGG16 model, while the AlexNet model using the training 
from the scratch approach achieved an $81.34 \%$ success rate. In terms of the $80-20$ separation value of training/test clusters, the highest accuracy score among the deep models was achieved with a 99.62\% rate by the VGG16 architecture based on the fine-tuning approach. Confusion matrices of the highest performance among deep models based on the fine-tuning and training from the scratch approaches are given in Figure 9.

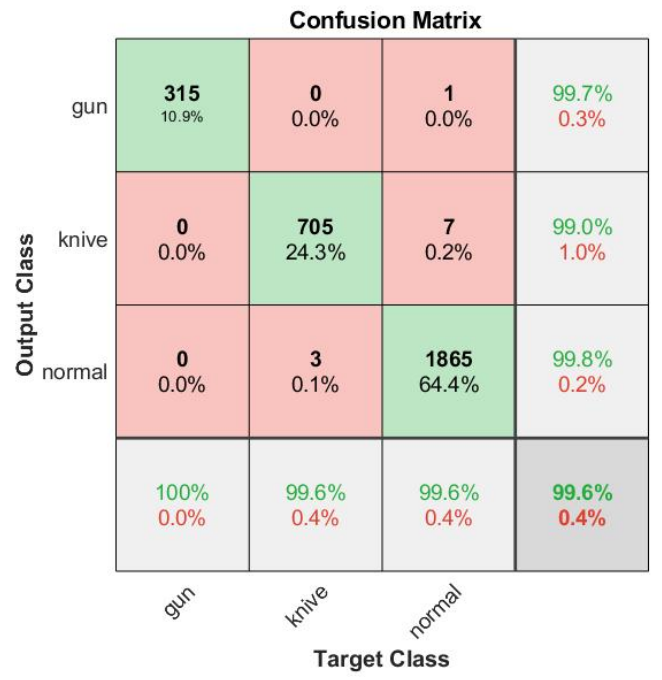

(a)

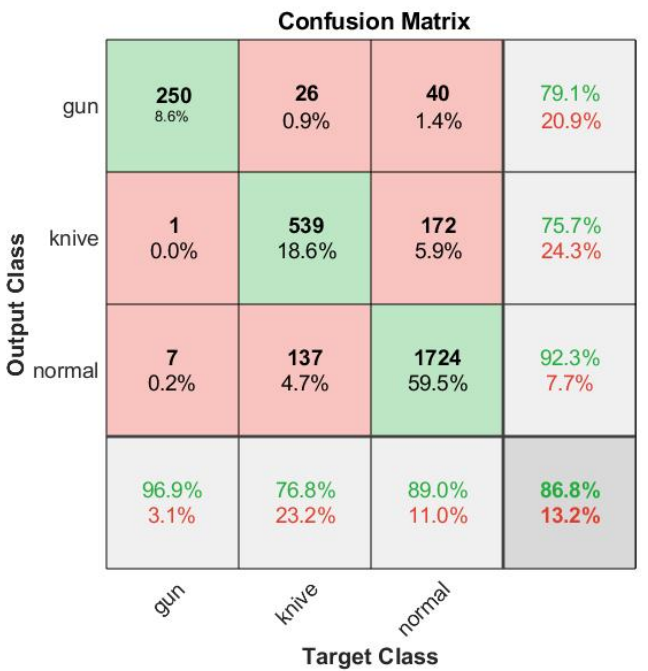

(b)

Figure 9. Confusion Matrices of proposed deep model based approaches to 3-class, a) Fine-tuning, b) Training from scratch.

As a result, it has been observed that the fine-tuning approach provides higher performance than the training from the scratch approach for pre-trained deep models. Besides, according to the results obtained from all experimental studies, the highest accuracy among deep models based on the finetuning method was obtained with the VGG16 model, while the AlexNet model got the highest score by using the training from scratch approach.

\section{Discussion}

Nowadays, most offensive actions are committed with criminal tools. One of the most effective ways of preventing malignant acts is to observe and detect the weapons in advance through security cameras. Many studies in the literature deal with the classification of criminal instruments such as guns and knives utilizing visuals containing offensive images. These studies are detailed in Table 4.

Table 4 Comparison of the proposed approach with previous studies

\begin{tabular}{|l|l|c|c|c|}
\hline References & Methods & $\begin{array}{c}\text { Number of } \\
\text { Classes }\end{array}$ & $\begin{array}{c}\text { Number of } \\
\text { Images }\end{array}$ & $\begin{array}{c}\text { Accuracy } \\
\text { (\%) }\end{array}$ \\
\hline$[32]$ & Fine-tuning based LeNet & 3 & 12,000 & 99 \\
\hline$[33]$ & $\begin{array}{l}\text { Visual vocabularies and deep } \\
\text { features }\end{array}$ & 4 & 1,950 & 95 and above \\
\hline$[2]$ & Alexnet+SVM & 2 & 2,000 & 95 \\
\hline$[15]$ & Fuzzy classification model & 2 & 12,899 & 86 \\
\hline$[34]$ & VGGNet & 3 & 5,504 & 98.41 \\
\hline \multirow{2}{*}{ The current study } & Fine-tuning based VGG16 & 2 & 12,899 & 99.73 \\
\cline { 2 - 5 } & & 3 & 14,481 & 99.62 \\
\hline
\end{tabular}

In Table 4, the accuracy scores of previous studies using knives, guns, and ordinary images are given. In these researches, generally, deep-learning-based models were used. In Table 4, it is observed that the 
current study has used more visuals than other works. As a result, it has been observed that the proposed research achieved a higher accuracy score than previous studies.

\section{Conclusion}

This study assessed the performances of deep convolutional neural networks based on different approaches for the classification of criminal tools. For this purpose, pre-trained AlexNet, VGG16, and VGG19 models were used. The performances of these models were calculated using Fine-tuning and Training from scratch approaches. As a result of extensive experimental studies, it was clearly observed that nearly $100 \%$ accuracy was obtained using the learned weights of deep architectures in the detection of crime tools. As a result, the study has observed that offensive weapons could be detected with security cameras using learned weights of deep architectures. In future studies, we are planning to detect videobased criminal devices using a deep neural network.

\section{References}

[1] "Umut Vakfi - Türkiye Silahlı Şiddet Haritas1 2019". [Online]. Available: http://umut.org.tr/umut-vakfi-turkiye-silahli-siddet-haritasi-2019/ [Accessed: 02-January-2021].

[2] S. B. Kibria and M. S. Hasan, "An analysis of Feature extraction and Classification Algorithms for Dangerous Object Detection,” In 2017 2nd International Conference on Electrical \& Electronic Engineering (ICEEE), pp. 1-4, 2017.

[3] R. Kanehisa and A. Neto, "Firearm Detection using Convolutional Neural Networks", [Online]. Available: https://www.scitepress.org/Link.aspx?doi=10.5220/0007397707070714/ [Accessed: 24-January-2021].

[4] M. Grega, A. Matiolański, P. Guzik, and M. Leszczuk, "Automated Detection of Firearms and Knives in a CCTV Image,” Sensors, vol. 16, no 1, pp. 47, 2016.

[5] J. L. Salazar González, C. Zaccaro, J. A. Álvarez-García, L. M. Soria Morillo, and F. Sancho Caparrini, "Real-time gun detection in CCTV: An open problem," Neural networks, vol. 132, pp. 297-308, 2020.

[6] G. K. Verma and A. Dhillon, “A Handheld Gun Detection using Faster R-CNN Deep Learning,” Proceedings of the 7th International Conference on Computer and Communication Technology, New York, NY, USA, pp. 84-88, 2017.

[7] R. K. Tiwari, and G. K. Verma, "A computer vision based framework for visual gun detection using harris interest point detector,” Procedia Computer Science, vol. 54, pp. 703-712, 2015.

[8] M. Kmieć, A. Głowacz, and A. Dziech, "Towards robust visual knife detection in images: active appearance models initialised with shape-specific interest points," In International Conference on Multimedia Communications, Services and Security, Berlin, Heidelberg, 2012, pp. 148-158, doi: 10.1007/978-3-642-30721-8_15.

[9] Castillo, S. Tabik, F. Pérez, R. Olmos, and F. Herrera, "Brightness guided preprocessing for automatic cold steel weapon detection in surveillance videos with deep learning," Neurocomputing, vol. 330, pp. 151-161, 2019.

[10] M. M. Fernandez-Carrobles, O. Deniz, and F. Maroto, "Gun and Knife Detection Based on Faster R-CNN for Video Surveillance,” Pattern Recognition and Image Analysis, Cham, pp. 441-452, 2019.

[11] H. Jain, A. Vikram, Mohana, A. Kashyap, and A. Jain, "Weapon Detection using Artificial Intelligence and Deep Learning for Security Applications," 2020 International Conference on Electronics and Sustainable Communication Systems (ICESC), pp. 193-198, 2020.

[12] R. Olmos, S. Tabik, and F. Herrera, "Automatic handgun detection alarm in videos using deep learning,” Neurocomputing, vol. 275, pp. 66-72, 2018.

[13] R. Olmos, S. Tabik, A. Lamas, F. Pérez-Hernández, and F. Herrera, “A binocular image fusion approach for minimizing false positives in handgun detection with deep learning," Inf. Fusion, no. 49, pp. 271-280, 2019. 
[14] F. Pérez-Hernández, S. Tabik, A. Lamas, R. Olmos, H. Fujita, and F. Herrera, “Object Detection Binary Classifiers methodology based on deep learning to identify small objects handled similarly: Application in video surveillance,” Knowl.-Based Syst., vol. 194, pp. 105590, 2020.

[15] A. Matiolański, A. Maksimova, and A. Dziech, "CCTV object detection with fuzzy classification and image enhancement,” Multimed. Tools Appl., vol. 75, no. 17, pp. 10513-10528, 2016.

[16] M. Türkoğlu, K. Hanbay, I. S. Sivrikaya, and D. Hanbay, "Derin Evrişimsel Sinir Ağ1 Kullanılarak Kayısı Hastalıklarının Sinıflandırılması," Bitlis Eren Üniversitesi Fen Bilimleri Dergisi, vol. 9, no. 1, pp. 334-345, 2021.

[17] "Introduction to Convolutional Neural Networks", 2018. [Online]. Available: https://rubikscode.net/2018/02/26/introduction-to-convolutional-neural-networks/ [Accessed: 03-January-2021].

[18] M. Turkoglu, "COVIDetectioNet: COVID-19 diagnosis system based on X-ray images using features selected from pre-learned deep features ensemble,” Applied Intelligence, pp. 1-14, 2020.

[19] K. Kayaalp, and S. Metlek, "Classification of robust and rotten apples by deep learning algorithm," Sakarya University Journal of Computer and Information Sciences, vol. 3, no. 2, pp. 112-120, 2020.

[20] I. Ozsahin, and D. U. Ozsahin, "Neural network applications in medicine,” In Biomedical Signal Processing and Artificial Intelligence in Healthcare, Academic Press, pp. 183-206, 2020.

[21] M. Türkoğlu, and D. Hanbay, "Plant disease and pest detection using deep learning-based features,” Turkish Journal of Electrical Engineering \& Computer Sciences, vol. 27, no. 3, pp. 1636-1651, 2019.

[22] H. Ahmetoğlu, and R. Daş, "Derin Öğrenme ile Büyük Veri Kumelerinden Saldırı Türlerinin Sinıflandırılmas1," In 2019 International Artificial Intelligence and Data Processing Symposium (IDAP), pp. 1-9, 2019,

[23] R. Daş, B. Polat, and G. Tuna, "Derin Öğrenme ile Resim ve Videolarda Nesnelerin Tanınmas1 ve Takibi,” Firat Üniversitesi Mühendislik Bilimleri Dergisi, vol. 31, no. 2, pp. 571-581, 2019.

[24] D. Şengür, and S. Siuly, "Efficient approach for EEG-based emotion recognition," Electronics Letters, vol. 56, no. 25, pp.1361-1364, 2020.

[25] A. Krizhevsk, I. Sutskever, and G. E. Hinton, "Imagenet classification with deep convolutional neural networks,” In: Advances in Neural Information Processing Systems, pp. 1097-1105, 2012.

[26] F. Doğan, and I. Türkoğlu, "Comparison of Leaf Classification Performance of Deep Learning Algorithms," Sakarya University Journal of Computer and Information Sciences, vol. 1, pp. 1021, 2018.

[27] M. Uçar, and E. Uçar, "Computer-aided detection of lung nodules in chest X-rays using deep convolutional neural networks," Sakarya University Journal of Computer and Information Sciences, vol. 2, no. 1, pp. 41-52, 2019.

[28] K. Simonyan, and A. Zisserman "Very deep convolutional networks for large-scale image recognition,” arXiv preprint, arXiv:1409.1556, 2014.

[29] D. Akgün, "An Evaluation of VGG16 Binary Classifier Deep Neural Network for Noise and Blur Corrupted Images," Sakarya University Journal of Computer and Information Sciences, vol. 3, no. 3, pp. 264-271, 2020.

[30] E. Erdem, and T. Aydın, "Detection of Pneumonia with a Novel CNN-based Approach," Sakarya University Journal of Computer and Information Sciences, vol. 4, no. 1, pp. 26-34, 2021.

[31] M. Turkoglu, O. F. Alcin, M. Aslan, A. Al-Zebari, and A. Sengur, "Deep rhythm and long short term memory-based drowsiness detection.," Biomedical Signal Processing and Control, vol. 65, pp. 102364, 2021.

[32] M. U. Salur, İ. Aydın, and M. Karaköse, "Gömülü Derin Öğrenme ile Tehdit İçeren Nesnelerin Gerçek Zamanda Tespiti,” Dicle Üniversitesi Mühendislik Fakültesi Mühendislik Dergisi, vol. 10, no. 2, pp. 497-509, 2019.

[33] D. Mery, E. Svec, M. Arias, V. Riffo, J. M. Saavedra, and S. Banerjee, "Modern computer vision techniques for x-ray testing in baggage inspection," IEEE Transactions on Systems, Man, and Cybernetics: Systems, vol. 47, no. 4, pp. 682-692, 2016. 
Sakarya University Journal of Computer and Information Sciences

A $\breve{g} d a s ̧$ et al.

[34] N. Dwivedi, D. K. Singh, and D. S. Kushwaha, "Weapon Classification using Deep Convolutional Neural Network,” In 2019 IEEE Conference on Information and Communication Technology, pp. 1-5, 2019. 